\title{
Maintenance treatment with infliximab for the management of Crohn's disease in adults
}

\author{
Renato Caviglia \\ Ivo Boškoski \\ Michele Cicala \\ Department of Digestive Diseases, \\ Campus Bio-Medico University \\ of Rome, Rome, Italy
}

\begin{abstract}
Crohn's disease (CD) is a chronic, relapsing disease, the continuous cycle of which deeply affects the long-term course which, eventually, leads to fibrosis and development of transmural complications. It is well known that $\mathrm{CD}$ is an immune-mediated clinical condition and that tumor necrosis factor- $\alpha$ (TNF- $\alpha$ ) plays a fundamental role in the pathogenesis of the disease. Current clinical guidelines recommend that patients with mild to moderate active CD should be treated initially with corticosteroids. Although this approach is effective in inducing remission, some patients may become dependent on, or refractory to, these drugs in the long term, thus increasing the risk of developing steroid-related adverse effects. A recent Cochrane systematic review established that infliximab (IFX) is effective in inducing remission in patients with CD. Although only a few published studies have assessed IFX for the maintenance of remission in the long term, there is evidence that IFX is superior to placebo in sustaining clinical remission and fistula healing; moreover, corticosteroid-sparing effects have been demonstrated. IFX is associated with the formation of antibodies to IFX which can lead to infusion reactions and shorter duration of response, but when comparing episodic vs scheduled maintenance treatment, the latter appears to sensibly reduce immunogenicity, thus offering improved efficacy and tolerance. The final point to consider is the best time to introduce IFX in the therapeutic algorithm of CD. Early use of IFX has been suggested to be more effective than late, and may potentially change the natural history of the disease. Effective induction and maintenance therapy with IFX is the only means with which to maintain long-lasting clinical and mucosal remission which, in turn, may modify the long-term course of the disease. Furthermore, when treating inflammatory bowel disease patients with IFX, an appropriate risk-benefit balance has to be taken into consideration, because the precise risk of serious adverse events associated with anti-TNF treatment in CD remains to be fully elucidated.
\end{abstract}

Keywords: inflammatory bowel disease, Crohn's disease, infliximab therapy, steroid sparing, tumor necrosis factor- $\alpha$

\section{Introduction}

Crohn's disease (CD), an inflammatory disorder which can involve any part of the gastrointestinal tract, is characterized by transmural damage of the bowel wall. ${ }^{1}$ The incidence of CD is approximately 5 to 10 new cases per 100,000 individuals/year. ${ }^{2}$ However, the incidence has been progressively increasing in Europe and North America. Estimated CD prevalence in North America ranges from 26.0 to 198.5 cases per 100,000 persons, which means 400,000 to 600,000 CD patients in North America alone. ${ }^{3,4}$ The pathogenesis of CD remains to be fully elucidated, but it is presumed to occur through a combination of three essential co-factors: host susceptibility, intestinal microflora, and mucosal immunity, the combined effect of which is sustained activation and uncontrolled response of the mucosal immune system against the normal commensal microbiota. ${ }^{5}$ In normal conditions, the mucosal immune system is in a constant state of "controlled inflammation". Homeostasis is achieved by a balance between $\mathrm{T}$ cell activation after antigen presentation and apoptosis. ${ }^{6} \mathrm{CD} 4+\mathrm{T}$-helper 1 
(Th 1) lymphocytes from patients with $\mathrm{CD}$ are resistant to the induction of apoptosis by a variety of stimuli. ${ }^{5}$ Moreover, the excessive activation of mucosal T cells, which is amplified and perpetuated by the increased release of pro-inflammatory cytokines, such as interferon $\gamma$, tumor necrosis factor- $\alpha$ (TNF- $\alpha$ ) and interleukin-12, by the intestinal lamina propria mononuclear cells, leads to transmural tissue damage, which is the pathologic characteristic of CD. ${ }^{7}$ The pro-inflammatory cytokine TNF- $\alpha$, appears to play a pivotal role in the pathogenesis of mucosal inflammation, mediating the inflammatory cascade in CD. ${ }^{8}$ TNF- $\alpha$ is mainly produced by monocytes and macrophages, although many other cells of the innate and adaptive immune system produce significant amounts of this cytokine ${ }^{8}$ moreover, several studies have demonstrated increased concentrations of TNF- $\alpha$ in blood, mucosa and stool from CD patients, thus making TNF- $\alpha$ a rational target in the treatment of active CD. ${ }^{9}$ In many patients, CD may be refractory to conventional treatment such as corticosteroids, enteral nutrition and immunomodulators (eg, azathioprine [AZA], 6-mercaptopurine [6-MP] and methotrexate [MTX]); ${ }^{10,11}$ on a long-term basis, some patients may become dependent on corticosteroids, thus increasing the risk of developing steroid-related adverse effects. ${ }^{12}$ Moreover, as shown by Cosnes et al, despite the increased use of immunosuppressants over the years, the need for surgical intervention in CD patients has remained high over the past few decades. ${ }^{13}$ In these clinical situations, it is important that other treatment options be considered. Over recent years, a growing number of reports have suggested that TNF- $\alpha$ blocking agents may be effective for inducing and mainting remission in CD. Many biologic compounds targeting TNF- $\alpha$ have been developed: the monoclonal antibody infliximab (IFX), a chimeric mouse/ human immunoglobulin (Ig) G1 anti-TNF- $\alpha$, was the first biologic agent to be used in the treatment of inflammatory bowel disease; the fully human IgG1 antibody adalimumab; the humanized Fab antibody fragment CDP-870; and etanercept and onercept, TNF- $\alpha$ receptor fusion proteins that bind to two specific transmembrane receptors of TNF- $\alpha$ (P75 and P55). ${ }^{14,15}$ Short-term studies have shown that use of these biologic compounds results in less need for surgical treatment and may alter the natural history of the disease through their ability to induce mucosal healing. ${ }^{16,17}$ Although IFX and adalimumab have been proven to be effective for 1 -year maintenance of steroid-free remission in patients with CD who respond to induction therapy, available clinical data for certolizumab pegol showed a significant rate of response and remission at week 26, only. Currently, the US Food and Drug Administration (FDA) approves all three biologic compounds for the treatment of CD, and the European Medicines Agency (EMEA) approves IFX and adalimumab but not certolizumab pegol. IFX was the first biologic compound to be used in the treatment of moderate to severe active $\mathrm{CD}$ and, therefore, most of the clinical experience on anti-TNF drugs in CD comes from this drug. Although the efficacy of a therapeutic strategy consisting of a loading dose of 3 IFX infusions and, thereafter, every 8 weeks, is supported by several placebo-controlled studies, very few data are available on the use of IFX for $>12$ months or $>8$ doses in active CD. ${ }^{16,18}$ The present review summarizes clinical data on long-term IFX treatment in CD. The literature search focused on papers published from 1999 to 2008. Abstracts from congress proceedings (Digestive Disease Week [DDW] United European Gastroenterology Week [UEGW] and European Crohn's and Colitis Organization [ECCO]) were also included.

\section{Infliximab}

\section{Structure and complex formation with TNF}

TNF- $\alpha$ originates as a membrane-anchored precursor (proTNF) which is cleaved by the metalloproteinase TNF- $\alpha-$ converting enzyme (TACE), to then be secreted as a $17-\mathrm{kDa}$ soluble protein. TNF- $\alpha$ exerts its effects through binding either one of the TNF- $\alpha$ receptors (TNFRs), TNFR1 or TNFR2. ${ }^{19}$ IFX is a chimeric monoclonal Ig G1 (75\% human and $25 \%$ murine) anti-TNF- $\alpha$. IFX is able to bind soluble and membrane-bound TNF, and in vitro data suggest that it can induce T-cell apoptosis via complement activation or antibody-dependent cell cytotoxicity. ${ }^{19}$ Besides its action on immune and epithelial cells, TNF- $\alpha$ plays a crucial role in regulating cell adhesion molecule expression by intestinal endothelium and fibroblasts. Danese et al recently reported that CD patients undergoing IFX treatment down-regulate the expression levels of the vascular cell adhesion molecule (VCAM-1) and CD40 on the intestinal mucosal endothelium, thus inhibiting T-cell recruitment. ${ }^{20}$ In addition to TNF- $\alpha$ blockade and apoptosis induction, IFX exerts a wide spectrum of anti-inflammatory activities. For instance, IFX reduces the circulating levels of basic fibroblast growth factor (bFGF) and vascular endothelial growth factor (VEGF). ${ }^{21}$ These two cytokines, proposed as markers of fibrosis and angiogenesis, are typically over-expressed in CD.

\section{Efficacy profile of long-term use of IFX in CD}

At present, biologic therapy with IFX is used in CD patients with fistulizing disease or who have not shown 
a good response to conventional agents. These include corticosteroid-dependent patients and/or those unresponsive/ intolerant to immunosuppressants (AZA/6-MP and MTX). Several randomized clinical trials (RCTs) have shown that IFX is effective in the treatment of clinically active CD patients, showing excellent results at short-term evaluation. ${ }^{16,17}$ However, although many CD patients have been treated with IFX on a long-term basis, to date only 4 RCTs evaluating IFX for the maintenance of remission in CD patients have been published (Table 1). ${ }^{22-25}$ In 1999, Rutgeerts et al published the results of the first RCT aimed at evaluating the efficacy and safety of repeated infusions with IFX 10 $\mathrm{mg} / \mathrm{kg}$ in patients showing a clinical response to an initial dose of IFX..$^{22}$ The 73 patients enrolled with moderate to severe CD came from a cohort of patients who had shown a clinical response to IFX at 4 weeks in the earlier study of Targan et $\mathrm{al}^{26}$ All patients, received 4 scheduled infusions every 8 weeks and were evaluated for efficacy and safety at 4-week intervals. At week 44, 8 weeks after the last infusion, $52.9 \%$ of the IFX-treated patients were in remission with only $20.0 \%$ of placebo $(\mathrm{p}=0.013)$. The ACCENT I trial (A Crohn's disease Clinical trial Evaluating IFX in a New longterm Treatment regimen) was the largest randomized, controlled clinical study designed to assess the efficacy of repeated infusions (on scheduled timing) of IFX in patients who had shown an initial response after the first infusion. ${ }^{23}$ Of the 580 patients enrolled with moderate to severe $\mathrm{CD}$ (Crohn's disease activity index [CDAI] between 220 and 440), 573 patients were started on IFX $5 \mathrm{mg} / \mathrm{kg}$; 335 (58\%) were responders at week 2 . The 335 responders were then randomized to receive placebo, $5 \mathrm{mg} / \mathrm{kg}$ maintenance regimen, or $10 \mathrm{mg} / \mathrm{kg}$ maintenance regimen. At week 54 , approximately 3 times as many patients ( $29 \%$ vs $9 \%$ ) on IFX vs placebo had maintained clinical remission. This study showed for the first time that the benefits of IFX in CD could be maintained, over the long term in patients treated with systematic maintenance therapy. In order to evaluate the efficacy of regularly scheduled retreatment vs episodic retreatment with IFX for maintenance of remission in patients with $\mathrm{CD}$, Rutgeerts et al performed a post hoc analysis on all patients who entered the ACCENT I study. ${ }^{27}$ This study evaluated the impact of the different treatment strategies on important outcome data such as mucosal healing, hospitalizations, and surgery. The results showed that regularly scheduled treatment with $5 \mathrm{mg} / \mathrm{kg}$ and $10 \mathrm{mg} / \mathrm{kg}$ IFX led to a statistically significant $(\mathrm{p}<0.05)$ higher proportion of patients in remission at weeks 10, 14, 22, and 46 compared with the episodic treatment group. Furthermore, $44 \%$ of patients in the regularly

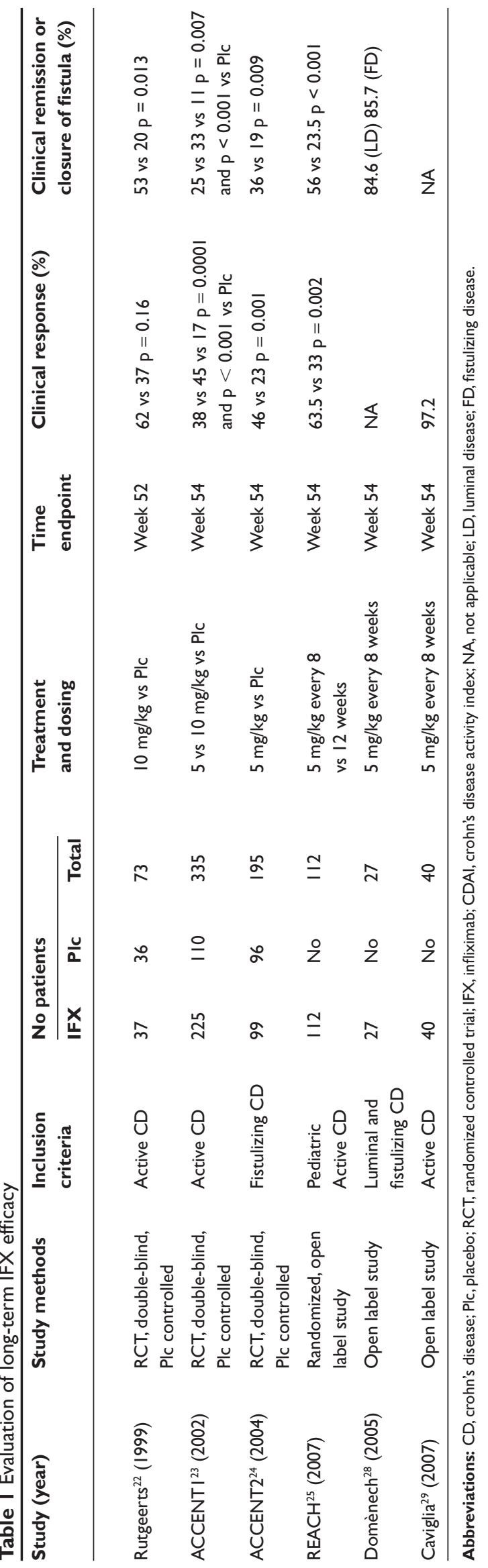


scheduled treatment groups had mucosal healing at week 54 compared with $18 \%$ in the episodic treatment group $(\mathrm{p}<0.014)$. Moreover, fewer CD-related hospitalizations occurred over the 54-week period in patients undergoing systematic maintenance therapy ( 24 events per 100 patients) than in patients treated episodically (38 events per 100 patients; $\mathrm{p}=0.023$ ). Only one randomized, double-blind, controlled study evaluated the long-term efficacy of IFX as maintenance treatment in patients with fistulizing $C D$. In the ACCENT II trial, 306 patients affected by fistulizing CD were initially treated with IFX $5 \mathrm{mg} / \mathrm{kg}$ at weeks 0,2 , and 6 , and responders were subsequently randomized to placebo or IFX $5 \mathrm{mg} / \mathrm{kg}$ at 8 -week intervals until the end of the study at week $54 .^{24}$ At week 14, of 306 patients, 195 (69\%) showed a response to the IFX induction therapy with closure of at least $50 \%$ of the fistulas. At week $54,39 \%$ of patients receiving maintenance IFX $5 \mathrm{mg} / \mathrm{kg}$ every 8 weeks and $19 \%$ of patients receiving placebo demonstrated complete closure of all fistulas ( $p<0.009)$. In a smaller, prospective, noncontrolled study, Domènech et al evaluated the clinical outcome of CD after induction of remission with 3 IFX infusions (23 patients with luminal disease) and after maintenance of remission with a 1 -year course of IFX every 8 weeks (27 patients with luminal and perianal disease). ${ }^{28}$ After the induction regimen, only 5 patients had a partial response and all relapsed in the first 2 months after the third IFX infusion. Among those patients with a complete response (18/23), after the induction treatment, 10 patients were observed at followup, with a median time of 33 months (range 8-54), and only one of them relapsed during this period (at 44 months). Only 5 out of $18(27.7 \%)$ patients who achieved complete remission relapsed within the first 6 months of follow-up. In contrast, early relapse in perianal disease was observed after drug withdrawal, even in patients with a long-term complete response while on IFX. Of the 14 patients with fistulizing CD who underwent a long-term scheduled regimen, 9 (64\%) relapsed after a mean time of $5.2 \pm 5.5$ months (range $2-18$ ). A retrospective analysis of data on the efficacy of long-term therapy with IFX in CD patients treated with a scheduled regimen has recently been published (Table 1). ${ }^{29}$ The medical charts of 50 patients (40 CD) who, after a loading dose of 3 IFX infusions, received scheduled retreatment every 8 weeks as maintenance protocol, were reviewed. In CD patients, median duration of treatment was 27 (range 4-64) months. Overall, 32 (80\%) CD patients showed a sustained clinical response or remission throughout the maintenance period. Three CD patients shortened the interval between infusions. Eight $(20 \%)$ CD patients underwent surgery on account of disease flare-up. Nine out of 29 CD patients who discontinued scheduled IFX treatment were still relapse-free after a median of 16 (range, 5-30) months after the last infusion. Recently, at the 3rd European Crohn's and Colitis Organisation Meeting, Schnitzler et al presented a retrospective analysis on a series of 614 consecutive CD patients treated long term with IFX, with a median follow-up of 55 (interquartile range [IQR $] 27-83$ ) months. ${ }^{30}$ Scheduled treatment was associated with less need for hospitalization (50/194, 26\%, median hospitalization 1 [IQR 1-2], median follow-up 1.91 years [IQR 0.92-3.42]) with respect to the episodic strategy $(165 / 353,47 \%$, median hospitalization 2 [IQR 1-4], median follow-up 6.05 years [IQR 4.24-7.27]) $(\mathrm{p}<0.0001)$; moreover, patients on a scheduled regimen from the start had a significantly better outcome than patients initially treated episodically (log rank: $p<0.0001)$. An important issue concerns the association of immunosuppressive drugs (AZA or MTX) in combination with IFX treatment. Although several published studies have addressed this topic, they show conflicting results. Lémann et al published results of an RCT carried out in order to evaluate the usefulness of IFX induction treatment combined with AZA or 6-MP, in steroid-dependent CD patients. ${ }^{31}$ Out of $113 \mathrm{CD}$ patients, 57 were randomized to receive IFX $5 \mathrm{mg} / \mathrm{kg}$ and 58 to receive placebo at weeks 0,2 , and 6 . A larger number of patients were in steroid-free remission at week 24 in the IFX group than in the placebo group (57\% vs $29 \%$; $=0.003$ ). At 56 weeks' follow-up, a similar trend was observed with a remission rate of $40 \%$ in IFX/AZA or 6-MP-treated patients compared to $22 \%$ placebo/AZA or 6 -MP-treated patients $(p=0.04)$. Van Assche et al evaluated the influence of discontinuation of immunosuppressives in CD patients in clinical remission with combination therapy (IFX plus AZA). ${ }^{32}$ Eighty patients were randomized either to continuation $(\mathrm{N}=40)$ or to withdrawal $(\mathrm{N}=40)$ of immunosuppressives, while all patients were on scheduled IFX maintenance treatment. When estimating the need to shorten the IFX dose intervals, no difference was found between the two groups of patients $(60 \%$ [95\% CI: 45\%-74\%] vs 55\% [95\% CI: 40\%-69\%], p=0.65). C-reactive protein (CRP) levels from week 8 through week 104 were significantly higher in the discontinuation group (median 2.8, IQR: 1.0-8.0) than in the continuation group (median 1.6, IQR: 1.0-5.6, p < 0.005). IFX trough levels from week 8 through week 54 were significantly higher in the continuation group than in the discontinuation group (median [IQR]: 2.87 [1.42-4.80] vs 1.65 [0.54-3.53] $\mu \mathrm{g} / \mathrm{mL}$, $\mathrm{p}<0.0001)$. The authors concluded that combined IFX and immunosuppressive treatment was not more efficacious than 
IFX monotherapy despite the finding of higher IFX concentrations and lower CRP levels in the combination regimen. On the contrary, at the 16th UEGW, Colombel et al presented the results of the SONIC (Study of Immunomodulator Naive patients in Crohn's Disease) trial, aimed at evaluating the efficacy of IFX vs AZA alone and vs the combination of both drugs, demonstrating the clear superiority of the combined treatment. ${ }^{33}$ This large multicenter study was carried out in more than 200 sites in the US, Israel, and Europe and comprised 508 patients naïve to both biologics and immunomodulators. The primary endpoint was steroidfree remission at week 26 and the secondary endpoint was endoscopic healing at week 56. At week 26, patients receiving IFX in combination with AZA showed the highest rate of remission (57\%) compared with IFX alone (44\%) or AZA alone $(30 \%)$. Considering mucosal healing at week 56 , endoscopic remission rates paralleled clinical remission rates in that patients on combination therapy scored the best, while those receiving IFX alone or AZA alone were second and third, respectively. If these data are confirmed in a full paper, they will have definite implications at least for the naïve patients, having shown that the early use of the combined treatment could be effective in decreasing short- and longterm complications such as surgery and hospitalizations. An interesting issue that should be taken into consideration is the effectiveness of early use of biologic therapy with combined immunosuppressants compared with conventional management (eg, corticosteroids) in patients with active CD (ie, top-down vs step-up strategy). D'Haens et al have, indeed, reported data from a comparison of topdown vs step-up therapy. ${ }^{34}$ The remission rate, defined as CDAI $<150$ without the use of steroids and without bowel resection, was significantly higher in patients in the early combined immunosuppression group than in those receiving conventional treatment, both at week 26 (60\% vs 36\%, $\mathrm{p}=0.0062)$ and at week $52(61.5 \%$ vs $42.2 \%, \mathrm{p}=0.0278)$. The authors concluded that remission rates in patients who received early combined immunosuppression were higher than those in patients receiving only corticosteroids. Finally, Schnitzler et al presented the results of a retrospective analysis on 614 consecutive $C D$ patients who underwent long-term IFX treatment over a median of 55 months followup, in order to assess the magnitude of loss of response and how to maintain clinical response by shortening of the interval between infusions, increasing dose of IFX, or a change from episodic to scheduled treatment every 8 weeks. ${ }^{35}$ Reduction of the interval between infusions was necessary in 108 patients $(19.7 \%)$, increase of the dose in 144 patients
$(26.3 \%)$, and increase of the dose plus a reduction of the interval in only 21 patients $(3.8 \%)$. Overall, in the total cohort of initial responders, only $21.6 \%(n=118)$ had to stop IFX treatment due to loss of response, despite interventions. The median time to switch to the every 8 weeks schedule in the episodic group was 26 months (IQR 12-47) and/or 6 infusions (IQR 4-10). The authors concluded that flexibility, both in dose and time-interval, may be useful to keep the disease under control in the long term. In conclusion, scheduled IFX treatment has proven to be an effective strategy in CD patients, for long-term maintenance of clinical remission; furthermore, long-lasting remission has been observed also after IFX withdrawal.

\section{Steroid-sparing effect}

For many years, corticosteroids have represented the standard therapy in inflammatory bowel disease, having been demonstrated to be efficacious in inducing a rapid clinical response, both in $\mathrm{CD}$ and ulcerative colitis; however, apart from their inability to maintain a long-lasting remission, the side effects after long-term use exceeded their clinical benefits. The steroid-sparing effect of IFX was another important finding emerging from several studies. In the ACCENT I trial, patients on maintenance IFX treatment were significantly more likely to remain in steroid-free remission at 54 weeks compared to those who received a single dose of IFX and were on placebo maintenance treatment $(29 \%$ vs $9 \% ; p=0.004) .{ }^{23}$ In a long-term IFX scheduled study, 10/31 (32.2\%) CD patients required concomitant steroid treatment during the maintenance period compared to $25 / 31(80.6 \%)$ patients on steroids at enrolment; moreover, in patients who were receiving corticosteroids, the median daily corticosteroid dose was reduced from $0.7 \mathrm{mg} / \mathrm{kg} /$ day at enrolment to $0.25 \mathrm{mg} / \mathrm{kg} / \mathrm{day} .{ }^{29}$ In the D'Haens et al study, which evaluated the long-term results of the two treatment strategies (top-down vs step-up approach), 17\% of patients in the conventional management group (eg, corticosteroids, followed in sequence by AZA and IFX) were still receiving corticosteroids compared with none of the patients in the top-down group (IFX and AZA), at 12 months. ${ }^{34}$ These data confirm the efficacy of an IFX scheduled treatment regimen in avoiding the well-known morbidity associated with longterm corticosteroid therapy.

\section{Evidence of mucosal healing}

ACCENT I was the first study to evaluate the effectiveness of long-term IFX treatment in inducing mucosal healing. Rutgeerts et al performed an endoscopic sub-study of the 
ACCENT I population, evaluating the effects of IFX on mucosal inflammation and mucosal healing. ${ }^{36}$ Endoscopic examinations were performed at baseline and at week 54 . The authors showed that a significantly higher proportion of week 2 responders in the scheduled maintenance group presented complete mucosal healing at week 54 compared with patients receiving the episodic treatment $(50 \%$ vs $7 \%, \mathrm{p}=0.007)$. A significantly greater improvement in the Crohn's Disease Endoscopic Index of Severity (CDEIS) score was observed with scheduled maintenance compared with episodic treatment at week $54(\mathrm{p}=0.026)$. Some abstracts recently presented at the DDW 2008 confirmed the effectiveness of long-term IFX treatment in inducing mucosal healing. In a long-term follow-up study by Schnitzler et $\mathrm{al}^{37}$ 614 consecutive $C D$ patients underwent long-term treatment with IFX between 1995 and 2007, with a median followup of 59 months; 8091 IFX infusions were administered. A total of 547 patients ( $89 \%$ ) showed a short-term response. In 388 patients, endoscopy was performed within a median of 1.61 months prior to first IFX infusion, and 263 patients with an initial response and further retreatment underwent endoscopy after a median of 11.05 months from starting IFX. In these patients, IFX treatment was scheduled in $33.5 \%$, episodic in $24.7 \%$ and episodic with switch to maintenance in $41.8 \%$. More than two thirds $(64.1 \%)$ were under concomitant immunomodulators and corticosteroids. Overall, 61.2\% of the 263 initial responders $(n=161)$ showed mucosal healing, with 106 patients having full mucosal healing (40.3\%) and $55(20.9 \%)$ improvement in the CDEIS after a median of 4 IFX infusions. Patients on scheduled treatment from the start were more likely to show mucosal healing than patients who were started on episodic treatment (67/88 vs 94/175, respectively, $\mathrm{p}<0.0001$, OR 2.75 [95\% CI 1.55-4.88]). Of the patients showing sustained clinical benefit until the end of follow-up, $65.9 \%$ presented mucosal healing compared to $50 \%$ of patients $(50 / 100)$ who withdrew from IFX due to loss of response or side effects $(\mathrm{p}<0.01)$. These results confirm the advantages of early use of IFX in the healing of mucosal ulcerations and that scheduled IFX therapy is more likely to result in mucosal healing than episodic therapy. Baert et al reported the results of a study aimed at evaluating the relevance of mucosal healing in $\mathrm{CD}$ in order to predict the clinical outcome in the following 2 years. ${ }^{38} \mathrm{~A}$ total of $133 \mathrm{CD}$ patients were randomized to treatment with combined immunosuppression, namely IFX and AZA or corticosteroids. Of the $133 \mathrm{CD}$ patients, 44 underwent ileocolonoscopy at 2 years. The Simple Endoscopic Score for CD (SES-CD) was used to evaluate endoscopic CD activity. Data were available in $42 / 44$ patients. A SES-CD score $=0$ at year 2 predicted stable clinical remission in the following 2-year period in $15 / 22$ patients (68\%) compared to $7 / 19(35 \%)$ patients with endoscopic activity (SES-CD 2-9) $(p=0.004)$. These results provide evidence that complete mucosal healing significantly increases clinical remission rates 2 years later. No significant differences in the use of medication were observed between the two groups during years 3 to 4 , probably due to the small number of patients studied $(\mathrm{p}=0.27)$.

\section{Issues requiring particular attention Immunogenicity}

The response of CD to IFX treatment is initially high. However, a loss of efficacy over time is observed in some cases. Several potential mechanisms may account for the progressive loss of response, among which the most important are drug-related mechanisms, including immunogenicity and tolerance to the drug. The development of antibodies against IFX may have a negative impact on the use of this compound in the clinical setting. Immunogenicity may be responsible for acute and delayed infusion reactions but may also cause a loss of clinical response. Baert et al in evaluating serum levels of antibodies to IFX (ATI) and correlating them with time to loss of response and infusion reactions ${ }^{39}$ (Table 2), found that concentrations of $8.0 \mu \mathrm{g} / \mathrm{mL}$ or greater before an infusion predicted a shorter duration of response (35 days vs 71 days in patients with concentrations $<8.0 \mu \mathrm{g} / \mathrm{mL}$; $\mathrm{p}<0.001)$ and a higher risk of infusion reactions ( $R R, 2.40$; 95\% CI, 1.65-3.66; $\mathrm{p}<0.001)$. In the ACCENT I trial, ATI were found in 64 out of 442 (14\%) patients: 41 (28\%) patients in the episodic-treated group compared to $23(15 \%)$ patients in the maintenance protocol (Table 2). ${ }^{23}$ The presence of ATI may also be correlated with infusion reaction; in fact, $38 \%$ of patients positive for antibodies to IFX had one or more infusion reactions compared with $24 \%$ of patients negative for antibodies to IFX, but $16 \%$ of infusions were associated with an infusion reaction in the ATI-positive patients compared with $8 \%$ of infusions in the ATI-negative patients. When considering the efficacy of the two treatment strategies (episodic vs scheduled), it should not be forgotten that intermittent or episodic dosing allows blood IFX concentrations to drop to undetectable levels while systematic scheduling regimens provide consistent therapeutic drug concentrations. Results emerging from the study by Hanauer et al demonstrated that the formation and concentrations of ATI were correlated with lower post-infusion serum IFX concentrations and with an increase in severe infusion reactions or serum sicknesslike reactions (Table 2). ${ }^{40}$ In this study, $573 \mathrm{CD}$ patients 


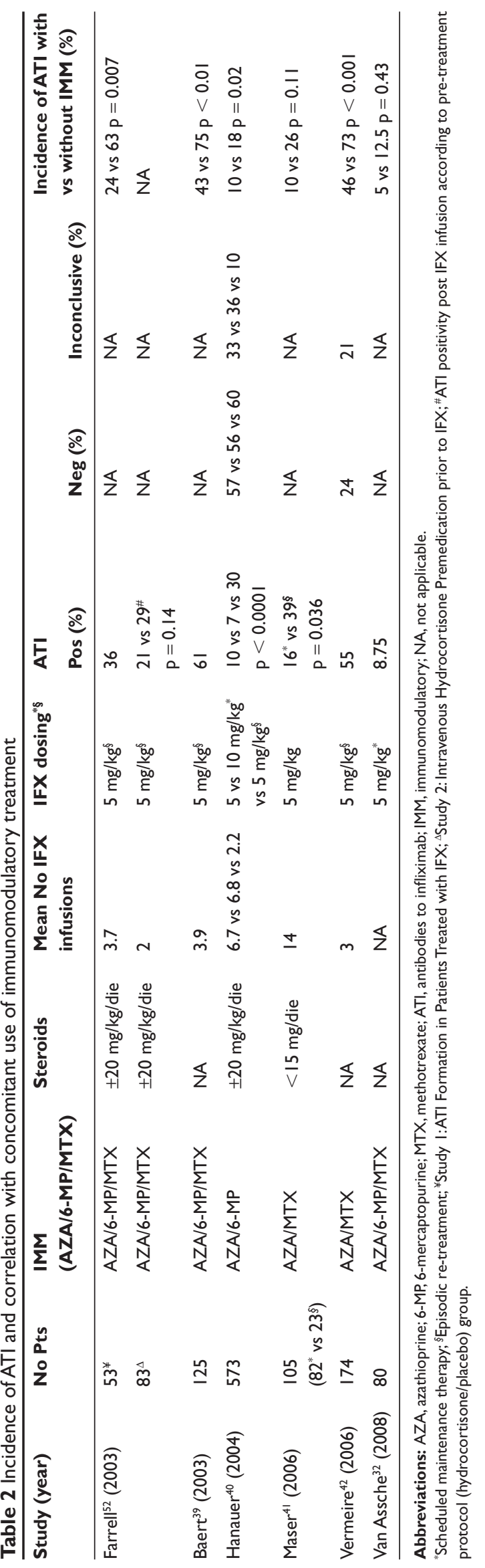

received $5 \mathrm{mg} / \mathrm{kg}$ of IFX (week 0 ) and were then randomly assigned to placebo (group I), $5 \mathrm{mg} / \mathrm{kg}$ IFX at weeks 2 and 6 and every 8 weeks thereafter until week 46 (group II), or $5 \mathrm{mg} / \mathrm{kg}$ IFX at weeks 2 and 6 , followed by $10 \mathrm{mg} / \mathrm{kg}$ thereafter (group III) as scheduled maintenance treatment. ATI were detected in $30 \%, 10 \%$ and $7 \%$ of groups I, II and III, respectively ( $<<0.0001)$. ATI were also associated with a $12 \%$ absolute increase in infusion reactions. The authors concluded that patients undergoing induction treatment followed by the scheduled maintenance regimen showed reduced ATI formation and a greater clinical benefit compared to those receiving a single dose followed by episodic retreatment. Similar results were reported by Maser et al who evaluated the effect of scheduled IFX infusion on antibody formation and their clinical significance in CD patients. ${ }^{41}$ ATI and trough serum IFX were measured in 105 patients with CD treated with IFX for induction of remission and followed by episodic retreatment $(n=23)$ or scheduled therapy at 6 - to 8 -week intervals $(n=82)$. ATI were present in $21 \%$ of patients, $25 \%$ of patients were ATI negative and $54 \%$ were antibody inconclusive. Antibody formation was higher after episodic compared to scheduled treatment (39\% vs $16 \%$; $<$ <.036). In the 105 patients evaluated, a positive relationship was found between serum concentration of IFX and clinical remission interval $\left(\mathrm{R}^{2}=0.61 ; \mathrm{p}<0.001\right)$. The rate of clinical remission was higher in patients with a detectable trough serum IFX compared with patients in whom serum IFX was undetectable, including those without antibodies $(82 \%$ vs $6 \%$; $<<0.001)$. Vermeire et al evaluated whether the concomitant use of immunosuppressive therapy (AZA or MTX) could decrease ATI formation, thus reducing the negative impact that these have on the clinical outcome (Table 2). ${ }^{42}$ Overall, $174 \mathrm{CD}$ patients treated with IFX episodic schedule were prospectively evaluated. Patients were divided into 3 groups: no immunosuppressives $(\mathrm{n}=59)$, concomitant MTX $(\mathrm{n}=50)$, and concomitant AZA $(n=65)$. ATI and IFX concentrations were measured before and 4 weeks after each infusion. The authors detected ATI in 55\% (96/174) of the patients. The concomitant use of immunosuppressive treatment was associated with a lower incidence of ATIs $(53 / 115 ; 46 \%)$ compared with patients not on concomitant immunosuppressive therapy (43/59; $73 \% ; \mathrm{p}<0.001)$. Patients not taking immunosuppressives had lower IFX levels (median $2.42 \mu \mathrm{g} / \mathrm{mL}$ ) 4 weeks after follow-up infusion than patients taking concomitant immunosuppressive therapy (median $6.45 \mu \mathrm{g} / \mathrm{mL})(\mathrm{p}=0.065)$, but there was no difference between MTX and AZA. In patients who developed significant ATI levels $(>8 \mu \mathrm{g} / \mathrm{mL})$ during 
follow-up, the IFX levels 4 weeks after the first infusion were retrospectively found to be significantly lower than in patients who did not develop ATI at follow-up.

\section{Stenosis}

Since CD is caused by a transmural inflammatory process, leading to symptomatic stenosis, the differentiation between a prevalent inflammatory and a fibromatous process is often difficult but is essential in order to adopt appropriate clinical management (ie, medical vs surgical treatment). Moreover, following reports of stricture and bowel obstruction occurring after IFX treatment, the concept that rapid mucosal healing with consequent excessive fibrogenesis, induced by this biologic agent, has given rise to concern that this treatment could lead to symptomatic intestinal stenosis, stricture, or obstruction (SSO). ${ }^{43,44}$ In order to shed further light on this issue, Lichtenstein et al analyzed data from the observational TREAT (Crohn's Therapy, Resource, Evaluation, and Assessment Tool) Registry and ACCENT I study in order to establish whether IFX treatment could increase the risk of intestinal stenosis. ${ }^{45}$ In the TREAT registry, 3,179 patients with 5,621 patient/years (pt/y) of follow-up were on IFX treatment and 3,111 patients with 4,651 pt/y of follow-up on other CD treatments. The authors showed that duration, severity and localization of the disease (ileal disease only) were significant predictors of symptomatic stenosis (hazard regression $[\mathrm{HR}]=1.02,95 \% \mathrm{CI} 1.00-1.04, \mathrm{HR}=2.35,95 \%$ CI $1.35-4.09, \mathrm{HR}=1.56,95 \%$ CI $1.04-2.36$, respectively) but, interestingly, new corticosteroid use (HR $=2.85,95 \%$ CI 1.23-6.57) was a significant predictor for stricture development. When considering IFX treatment, symptomatic stenosis or obstruction occurred in 88 IFX-treated patients compared to 49 patients who received other treatments only (1.95 events/100 pt/y vs 0.99 events/100 pt/y, p < 0.001, univariate regression analysis). But when data were adjusted for other factors, such as disease characteristics, neither previous nor new IFX use was significantly associated with stenosis development $(\mathrm{HR}=1.11,95 \%$ CI $0.72-1.73$ and $\mathrm{HR}=1.65,95 \%$ CI 0.74-3.70). Therefore, even if standard univariate analysis demonstrated a higher incidence of stricture development in IFX-treated patients, it is possible with multivariable analysis to separate the individual factor associated with development of SSO, thus suggesting that IFX treatment could have been proposed in CD patients with a more advanced stage of the disease, more likely presenting fibrostenotic disease per se. In the ACCENT I trial, a total of 573 patients were randomly assigned to receive either episodic treatment, or scheduled maintenance treatment with either IFX $5 \mathrm{mg} / \mathrm{kg}$ or $10 \mathrm{mg} / \mathrm{kg}$. Symptomatic stenosis was reported in $12(6.4 \%)$ patients who received episodic treatment and in $10(5.2 \%)$ and $13(6.7 \%)$ patients who received $5 \mathrm{mg} / \mathrm{kg}$ or $10 \mathrm{mg} / \mathrm{kg}$ IFX scheduled strategy, respectively $(p>0.05)$. No increase in the occurrence or severity of stenosis was observed despite different IFX dose exposure. An interesting study by Di Sabatino et al attempted to find a pathophysiologic explanation for the potential influence of IFX on intestinal fibrogenesis in $\mathrm{CD}$, by measuring serum levels of bFGF and VEGF, two cytokines known to promote repair of tissue damaged by inflammation, through modulation of fibroblast proliferation. ${ }^{21}$ The authors evaluated serum levels of bFGF and VEGF in $22 \mathrm{CD}$ patients, during and after 12 weeks of IFX treatment. Results showed that IFX can induce a reduction in serum bFGF and VEGF levels in almost all patients: $\mathrm{bFGF}(39.3 \pm 13.2 \mathrm{vs} 21.8 \pm 7.1 \mathrm{pg} / \mathrm{mL})$ and VEGF $(1003 \pm 227$ vs $486 \pm 180 \mathrm{pg} / \mathrm{mL}, \mathrm{p}<0.001)$ levels 2 weeks after the first infusion. Although these findings did not allow the authors to conclude that CD patients with symptomatic stenosis could be treated with IFX, they do suggest the possibility that IFX can be used to prevent the onset of fibrosis in patients not presenting a previous history of obstruction, or when the intestinal stenosis in CD results from a prevalent inflammatory component rather than a fibrotic one.

\section{Conclusions}

The results of years of intensive research aimed at a better understanding of the pathobiology of CD led to a new approach in the management of patients unresponsive/intolerant to standard therapies such as steroids and/or immunosuppressors. Biologic compounds have been developed in order to target specific molecules and/or pathways involved in CD pathogenesis. TNF- $\alpha$ is likely to be a key mediator in the inflammatory cascade playing a central role in mucosal inflammation, so, based on this rationale, antagonizing this cytokine has brought a dramatic change both in the patient's and the physician's perspective of CD treatment. IFX, the first anti-TNF biologic agent approved for the treatment of $\mathrm{CD}$, has been demonstrated to be effective both in inducing and maintaining clinical remission for at least 1 year (based on clinical trial data). ${ }^{22-25}$ These findings have been confirmed even in the pediatric population. Indeed, Hyams et al evaluated the efficacy of maintenance treatment with IFX in children with $\mathrm{CD}$, demonstrating that the scheduled administration of IFX every 8 weeks is able to keep the disease in remission for the long term. ${ }^{25}$ At 1 year follow-up, remission rate was $55.8 \%$. Although no controlled study 
data are available on the use of IFX in CD beyond 1 year, a growing body of evidence from IFX and other anti-TNF clinical studies appears to indicate the efficacy of long-term treatment as a disease-control strategy. After a decade of successful IFX treatment in CD patients, evolving evidence has offered some clues on the management of this drug in this setting. It appears evident that an induction regimen followed by scheduled maintenance treatment is superior to an ondemand strategy in order to obtain greater clinical benefit and long-lasting clinical remission which, in turn, may modify the long-term course of the disease. In addition, clinicians are focusing on new treatment strategies aimed at modulating the natural course of CD by using IFX at an early stage of the disease, before the disease itself has induced irreversible damage. Indeed, in rheumatoid arthritis, early intervention with combined immunosuppression (anti-TNF- $\alpha$ plus immunosuppressants) has been shown to prevent progressive joint destruction and improve long-term functional outcomes. ${ }^{46-48}$ In $\mathrm{CD}$, preliminary data from a clinical trial evaluating this early aggressive therapeutic approach showed that combined immunosuppression (IFX plus AZA) was more effective than conventional management (corticosteroids) in the induction of remission, reduction of corticosteroid use and in achieving mucosal healing. ${ }^{33,34}$ Moreover, patients who received early combined immunosuppression were much more likely to maintain mucosal healing after 2 years of treatment. ${ }^{34}$ Mucosal healing has now become a major goal of medical treatment in CD. Indeed, it was the objective of a sub-analysis of the ACCENT I study. ${ }^{36}$ In this trial, no patients showing endoscopic response to IFX therapy required hospitalization compared to $28 \%$ of those patients not achieving mucosal healing. Therefore, these results, together with those of Schnitzler et al support the evidence that IFX could substantially reduce patient-care costs for these patients and, hopefully, change the natural course of CD. ${ }^{37}$ Multiple clinical trials have shown that development of ATI is an important epiphenomenon of IFX treatment. ${ }^{39-42}$ In fact, ATI have been correlated with infusion reactions and loss of response. ${ }^{39,40}$ Various strategies have been studied in order to decrease ATI formation, but only systematic treatment, consisting of induction and scheduled maintenance, has proven effective in reducing ATI levels. ${ }^{40,41}$ The concomitant use of immunosuppressants and IFX administration has led to conflicting results about ATI formation. Another issue regarding the use of IFX in CD is whether there is a cause-effect relationship between IFX and intestinal strictures or whether it can be used in their presence. On this issue, although the paper by Lichtenstein et al showed that treatment with IFX does not appear to increase the risk of stricture development in patients with CD, these authors suggested that the use of IFX should be avoided in patients with obstructive symptoms or presenting a stricture, with a bowel retro-dilation. ${ }^{45}$ Despite substantial clinical evidence that IFX treatment may play a major role in the therapeutic algorithm in CD, some concerns about its long-term use remain. Safety concerns associated with long-term use of IFX, or the high cost of scheduled maintenance treatment, or possibly both, have led physicians to become reluctant to use this compound. ${ }^{49,50}$ To maximize treatment efficacy and minimize side effects, prospective studies aimed at predicting subgroups of patients who will develop an aggressive disease course (eg, young age at diagnosis [ $<40$ years], perianal disease, need of systemic steroids at first presentation) are mandatory in order to identify those CD patients who would benefit from an early and aggressive treatment strategy with biologic agents. ${ }^{51}$ Assigning these agents in the correct context for $\mathrm{CD}$ patients has become the future challenge for the clinician.

\section{Abbreviations}

ATI, antibodies to IFX; AZA, azathioprine; CD, Crohn's disease; IFX, infliximab (IFX); 6-MP, 6-mercaptopurine; TNF- $\alpha$, tumor necrosis factor- $\alpha$; Tb, tuberculosis; RCTs, randomized control trials.

\section{Disclosures}

None of the authors have conflicts of interest to disclose.

\section{References}

1. Fiocchi C. Inflammatory bowel disease: etiology and pathogenesis. Gastroenterology. 1998;115(1):182-205.

2. Loftus EV Jr, Sandborn WJ. Epidemiology of inflammatory bowel disease. Gastroenterol Clin North Am. 2003;31:1-20.

3. Rubin GP, Hungin AP, Kelly PJ, et al. Inflammatory bowel disease: epidemiology and management in an English general practice population. Aliment Pharmacol Ther. 2000;14:1553-1559.

4. Loftus EF Jr, Schoenfeld P, Sandborn WJ. The epidemiology and natural history of Crohn's disease in population-based patient cohorts from North America: a systematic review. Aliment Pharmacol Ther. 2002;16:51-60.

5. Peyrin-Biroulet L, Desreumaux P, Sandborn WJ, et al. Crohn's disease: beyond antagonists of tumour necrosis factor. Lancet. 2008;372(9632):67-81.

6. Cario E, Podolsky DK. Intestinal epithelial TOLLerance versus inTOLLerance of commensals. Mol Immunol. 2005;42(8):887-893.

7. Garrett WS, Lord GM, Punit S, et al. Communicable ulcerative colitis induced by T-bet deficiency in the innate immune system. Cell. 2007;131:33-45.

8. Hecht GA. Inflammatory bowel disease - live transmission. $N$ Engl $J$ Med. 2008;358(5):528-530.

9. Danese S. Mechanisms of action of infliximab in inflammatory bowel disease: an anti-inflammatory multitasker. Dig Liver Dis. 2008;(40 Suppl 2):S225-228 
10. Schreiber S. Medical treatment: an overview. In: Satsangi J. Inflammatory Bowel Diseases. Churchill Livingstone. Elsevier Limited; 2003. p. 297-301.

11. Lichtenstein GR, Abreu MT, Cohen R, et al. American Gastroenterological Association. American Gastroenterological Association Institute technical review on corticosteroids, immunomodulators, and infliximab in inflammatory bowel disease. Gastroenterology. 2006;130(3):940-987.

12. Faubion WA Jr, Loftus EV Jr, Harmsen WS, et al. The natural history of corticosteroid therapy for inflammatory bowel disease: a populationbased study. Gastroenterology. 2001;121:255-260.

13. Cosnes J, Nion-Larmurier I, Beaugerie L, et al. Impact of the increasing use of immunosuppressants in Crohn's disease on the need for intestinal surgery. Gut. 2005;54:237-241.

14. Hanauer S. Efficacy and safety of tumor necrosis factor antagonists in Crohn's disease: Overview of randomized clinical studies. Rev Gastroenterol Disord. 2004;3:S18-24.

15. Peyrin-Biroulet L, Deltenre P, de Suray N, et al. Efficacy and safety of tumor necrosis factor antagonists in Crohn's disease: metaanalysis of placebo-controlled trials. Clin Gastroenterol Hepatol. 2008;6(6):644-653.

16. Akobeng AK, Zachos M. Tumor necrosis factor-alpha antibody for induction of remission in Crohn's disease. Cochrane Database Syst Rev. 2004;(1):CD003574.

17. Rahimi R, Nikfar S, Abdollahi M. Do anti-tumor necrosis factors induce response and remission in patients with acute refractory Crohn's disease? A systematic meta-analysis of controlled clinical trials. Biomed Pharmacother. 2007;61:75-80.

18. Lichtenstein GR, Abreu MT, Cohen R, et al. American Gastroenterological Association. American Gastroenterological Association Institute technical review on corticosteroids, immunomodulators, and infliximab in inflammatory bowel disease. Gastroenterology. 2006;130(3):940-987.

19. Scallon BJ, Moore MA, Trinh H, et al. Chimeric anti-TNF-alpha monoclonal antibody cA2 binds recombinant transmembrane TNF-alpha and activates immune effector functions. Cytokine. 1995;7:251-259.

20. Danese S, Sans M, Scaldaferri F, et al. TNF-alpha blockade downregulates the $\mathrm{CD} 40 / \mathrm{CD} 40 \mathrm{~L}$ pathway in the mucosal microcirculation: a novel anti-inflammatory mechanism of infliximab in Crohn's disease. J Immunol. 2006;176:2617-2624.

21. Di Sabatino A, Ciccocioppo R, Benazzato L, et al. Infliximab downregulates basic fibroblast growth factor and vascular endothelial growth factor in Crohn's disease patients. Aliment Pharmacol Ther. 2004;19:1019-1024.

22. Rutgeerts P, D'Haens G, Targan S, et al. Efficacy and safety of retreatment with anti-tumor necrosis factor antibody (infliximab) to maintain remission in Crohn's disease. Gastroenterology, 1999;117:761-769.

23. Hanauer S, Feagan BG, Lichtenstein GR, et al. Maintenance infliximab for Crohn's disease: the ACCENT I randomised trial. Lancet. 2002;359:1541-1549.

24. Sands BE, Anderson FH, Bernstein CN, et al. Infliximab maintenance therapy for fistulizing Crohn's disease. $N$ Engl $J$ Med. 2004;350:876-885.

25. Hyams J, Crandall W, Kugathasan S, et al. REACH Study Group. Induction and maintenance infliximab therapy for the treatment of moderate-to-severe Crohn's disease in children. Gastroenterology. 2007;132(3):863-873.

26. Targan SR, Hanauer SB, van Deventer SJ, et al. A short-term study of chimeric monoclonal antibody $\mathrm{cA} 2$ to tumor necrosis factor alpha for Crohn's disease. Crohn's Disease cA2 Study Group. N Engl J Med. 1997;337(15):1029-1035.

27. Rutgeerts P, Feagan BG, Lichtenstein GR, et al. Comparison of scheduled and episodic treatment strategies of infliximab in Crohn's disease. Gastroenterology. 2004;126:402-13.

28. Domènech E, Hinojosa J, Nos $\mathrm{P}$, et al. Clinical evolution of luminal and perianal Crohn's disease after inducing remission with infliximab: how long should patients be treated? Aliment Pharmacol Ther. 2005;22(11-12):1107-1113.
29. Caviglia R, Ribolsi M, Rizzi M, et al. Maintenance of remission with infliximab in inflammatory bowel disease: efficacy and safety long-term follow-up. World J Gastroenterol. 2007;13(39):5238-5244.

30. Schnitzler F, Fidder H, Ferrante M, et al. Infliximab therapy for refractory Crohn's disease decreases the long-term need for bowel surgery. J Crohn's Colitis. 2008;2(1 Suppl A):001.

31. Lémann M, Mary JY, Duclos B, et al. Groupe d'Etude Therapeutique des Affections Inflammatoires du Tube Digestif (GETAID). Infliximab plus azathioprine for steroid-dependent Crohn's disease patients: a randomized placebo-controlled trial. Gastroenterology. 2006;130(4):1054-1061.

32. Van Assche G, Magdelaine-Beuzelin C, D'Haens G, et al. Withdrawal of immunosuppression in Crohn's disease treated with scheduled infliximab maintenance: a randomized trial. Gastroenterology. 2008;134(7):1861-1868.

33. Colombel JF, Rutgeerts P, Reinisch W, et al. SONIC: A randomized, double-blind, controlled trial comparing infliximab and infliximab plus azathrioprine to azathioprine in patients with Crohn's disease naive to immunomodulators and biologic therapy. Gut. 2008;57( Suppl II):A-1.

34. D'Haens G, Baert F, Van Assche G, et al. Early combined immunosuppression or conventional management in patients with newly diagnosed Crohn's disease: an open randomised trial. Lancet. 2008;371(9613):660-667.

35. Schnitzler F, Fidder H, Ferrante M, et al. Flexibility in interval and dosing of infliximab enables maintained response of patients with Crohn's disease. Gastroenterology. 2008;134(Suppl 1):A-658.

36. Rutgeerts $\mathrm{P}$, Diamond RH, Bala M, et al. Scheduled maintenance treatment with infliximab is superior to episodic treatment for the healing of mucosal ulceration associated with Crohn's disease. Gastrointest Endosc. 2006;63(3):433-442.

37. Schnitzler F, Fidder H, Ferrante M, et al. Maintenance Q8 therapy of Crohn's disease with infliximab is associated with endoscopic mucosal healing in the long-term. Gastroenterology. 2008;134(4 Suppl 1):A-133.

38. Baert F, Moortgat L, Van Assche G, et al. Mucosal healing predicts sustained clinical remission in early Crohn's disease. Gastroenterology. 2008;134(4 Suppl 1):A-640.

39. Baert F, Noman M, Vermeire S, et al. Influence of immunogenicity on the long-term efficacy of infliximab in Crohn's disease. $N$ Engl J Med. 2003;348(7):601-608.

40. Hanauer SB, Wagner CL, Bala M, et al. Incidence and importance of antibody responses to infliximab after maintenance or episodic treatment in Crohn's disease. Clin Gastroenterol Hepatol. 2004;2(7):542-553.

41. Maser EA, Villela R, Silverberg MS, et al. Association of trough serum infliximab to clinical outcome after scheduled maintenance treatment for Crohn's disease. Clin Gastroenterol Hepatol. 2006;4(10):1248-1254.

42. Vermeire S, Noman M, Van Assche G, et al. Effectiveness of concomitant immunosuppressive therapy in suppressing the formation of antibodies to infliximab in Crohn's disease. Gut. 2007;56(9):1226-1231.

43. Toy LS, Scherl EJ, Kornbluth A, et al. Complete bowel obstruction following initial response to IFX therapy for Crohn's disease: A series of a newly described complication. Gastroenterology. 2000;118:A2974.

44. Vasilopoulos S, Kugathasan S, Saeian K, et al. Intestinal strictures complicating initially successful IFX treatment for luminal Crohn's disease. Am J Gastroenterol. 2000;95:2503.

45. Lichtenstein GR, Olson A, Travers S, et al. Factors associated with the development of intestinal strictures or obstructions in patients with Crohn's disease. Am J Gastroenterol. 2006;101:1030-1038.

46. Goekoop-Ruiterman YP, De Vries-Bouwstra JK, Allaart CF, et al. Clinical and radiographic outcomes of four different treatment strategies in patients with early rheumatoid arthritis (the BeSt study): a randomized, controlled trial. Arthritis Rheum. 2005;52:3381-3390.

47. Breedveld FC, Weisman MH, Kavanaugh AF, et al. The PREMIER study: a multicenter, randomized, double-blind clinical trial of combination therapy with adalimumab plus methotrexate versus methotrexate alone or adalimumab alone in patients with early, aggressive rheumatoid arthritis who had not had previous methotrexate treatment. Arthritis Rheum. 2006;54:26-37. 
48. Smolen JS, van der Heijde DM, St Clair EW, et al. Predictors of joint damage in patients with early rheumatoid arthritis treated with high-dose methotrexate with or without concomitant infliximab: results from the ASPIRE trial. Arthritis Rheum. 2006;54:702-710.

49. Caviglia R, Boškoski I, Cicala M. Long-term treatment with infliximab in inflammatory bowel disease: safety and tolerability issues. Expert Opin Drug Saf. 2008;7(5):617-632.
50. Blonski W, Lichtenstein GR. Safety of biologic therapy. Inflamm Bowel Dis. 2007;13(6):769-796.

51. Beaugerie L, Seksik P, Nion-Larmurier I, et al. Predictors of Crohn's disease. Gastroenterology. 2006;130:650-656.

52. Farrell RJ, Alsahli M, Jeen YT, et al. Intravenous hydrocortisone premedication reduces antibodies to infliximab in Crohn's disease: a randomized controlled trial. Gastroenterology. 2003;124(4):917-924. 
